\title{
New project for precise neutron lifetime measurement at J-PARC
}

Naoki Nagakura ${ }^{1, a}$, Katsuya Hirota $^{2}$, Sei Ieki ${ }^{1}$, Takashi Ino $^{3}$, Yoshihisa Iwashita ${ }^{4}$, Masaaki Kitaguchi ${ }^{5}$, Ryunosuke Kitahara ${ }^{6}$, Jun Koga ${ }^{7}$, Kenji Mishima ${ }^{3}$, Aya Morishita ${ }^{7}$, Yusuke Nakano ${ }^{2}$, Hideyuki Oide ${ }^{8}$, Hiroki Okabe ${ }^{2}$, Hidetoshi Otono ${ }^{9}$, Yoshichika Seki ${ }^{10}$, Daiichiro Sekiba ${ }^{11}$, Tatsushi Shima ${ }^{12}$, Hirohiko Shimizu ${ }^{2}$, Naoyuki Sumi ${ }^{7}$, Hirochika Sumino ${ }^{13}$, Kaoru Taketani ${ }^{3}$, Tatsuhiko Tomita ${ }^{7}$, Hideaki Uehara ${ }^{7}$, Takahito Yamada ${ }^{1}$, Satoru Yamashita $^{14}$, Mami Yokohashi $^{2}$, and Tamaki Yoshioka ${ }^{9}$

${ }^{1}$ Department of Physics, Graduate School of Science, The University of Tokyo, Japan

${ }^{2}$ Department of Physics, Graduate School of Science, Nagoya University, Japan

${ }^{3}$ KEK, High Energy Accelerator Research Organization, Japan

${ }^{4}$ Institute for Chemical Research, Kyoto University, Japan

${ }^{5}$ Kobayashi-Maskawa Institute for the Origin of Particles and the Universe (KMI), Nagoya University, Japan

${ }^{6}$ Department of Physics, Graduate School of Science, Kyoto University, Japan

${ }^{7}$ Department of Physics, Graduate School of Science, Kyushu University, Japan

${ }^{8}$ Istituto Nazionale di Fisica Nucleare (INFN-Genova), Japan

${ }^{9}$ Research Center for Advanced Particle Physics (RCAPP), Kyushu University, Japan

${ }^{10}$ J-PARC Center, Japan Atomic Energy Agency, Japan

${ }^{11}$ Institute of Applied Physics, University of Tsukuba, Japan

${ }^{12}$ Research Center for Nuclear Physics (RCNP), Osaka University, Japan

${ }^{13}$ Department of Basic Science, Graduate School of Arts and Sciences, The University of Tokyo, Japan

${ }^{14}$ International Center for the Elementary Particle Physics (ICEPP), The University of Tokyo, Japan

\begin{abstract}
The decay lifetime of free neutrons $(\sim 880 \mathrm{~s})$ is an important parameter of the weak interaction and for Big Bang Nucleosynthesis. However, results of measurements currently show discrepancies depending on the method used. As most experiments nowadays employ ultra cold neutrons, we have developed a new cold-beam experiment which we perform at the Japan Proton Accelerator Research Complex. As a special feature, a polarized neutron beam is bunched by a spin flip chopper. A time projection chamber operated with $\mathrm{He}$ and $\mathrm{CO}_{2}$ gas, including a well-controlled amount of ${ }^{3} \mathrm{He}$, is used for detection of the beta-decays and simultaneous determination of the beam intensity. Using the data between 2014 and 2016, we evaluated our first, preliminary result of the neutron lifetime as $896 \pm 10$ (stat.) ${ }_{-10}^{+14}$ (sys.) s. We plan several upgrades to achieve our precision goal of $1 \mathrm{~s}$.
\end{abstract}

\section{Introduction}

Since the neutron was discovered by J. Chadwick in 1932 [1], many experiments have been conducted to measure its decay lifetime $\left(\tau_{n}\right)$. This is on one hand used as an input for calculations of final abundances of the light elements created in Big Bang Nucleosynthesis in the early universe [2]. In addition, $\tau_{n}$ is also used to evaluate the $V_{\text {ud }}$ element in the Cabbio-Kobayashi-Maskawa (CKM) matrix [3]. The neutron lifetime has been measured mainly by two independent methods: (1) storage of ultra cold neutrons (UCNs), (2) in-beam detection of charged decay particles. In the former, UCNs are stored in a chamber for a certain period of time. The neutron lifetime is evaluated by counting the remaining UCNs. In the latter, on the other hand, the cold neutron beam is injected into a decay volume from which one counts the charged decay particles. The average of the most recent results [4-8] obtained with the UCN method is $(879.6 \pm 0.6) \mathrm{s}$,

a e-mail: nagakura@icepp.s.u-tokyo.ac.jp while that deduced from the two most recent in-flight experiments $[9,10]$ using detection of decay protons is $(888.0 \pm 2.0) \mathrm{s}$. The $4 \sigma$ deviation of $8.4 \mathrm{~s}$ between these two averages is not understood yet. In order to solve this problem, and since the average value from all previous inbeam measurements is dominated by a single experiment [10], we have developed a new apparatus to perform an in-beam experiment using a different method. Here we report our first experiments carried out at the Japan Proton Accelerator Complex (J-PARC).

\section{Measurement principle}

A schematic view of the experimental setup is shown in Fig. 1. A spin flip chopper (SFC) fed with a beam of polarized neutrons creates bunches which enter a time projection chamber (TPC) wherein the number of neutron beta-decay events is counted. A small amount of well-calibrated ${ }^{3} \mathrm{He}$ gas allows us to detect neutrons using the reaction ${ }^{3} \mathrm{He}(n, p){ }^{3} \mathrm{H}$ in the TPC for absolute determination of the neutron particle flux. The length of 


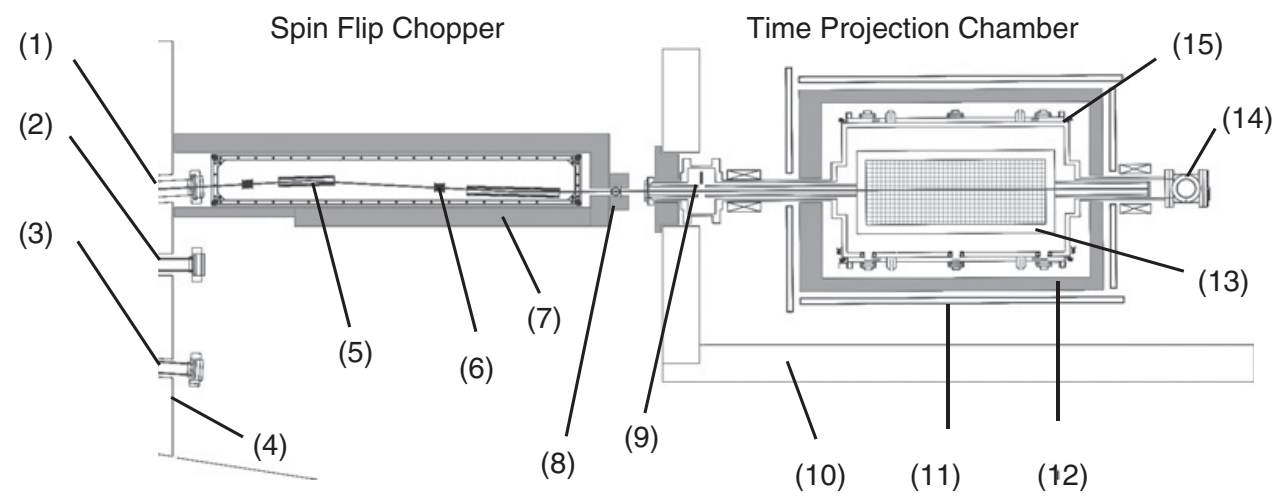

Figure 1. Schematic view of the experimental setup [11]: (1) exit of polarized beam branch, (2) exit of non-polarized beam branch, (3) exit of low-divergence beam branch of the NOP beamline, (4) lead shield, (5) magnetic super mirror, (6) radio frequency coil, $(7,8)$ lead shield, (9) neutron shutter (5 mm-thick 6LiF), (10) iron shield, (11) plastic scintillator, (12) lead shield, (13) TPC, (14) beam dump, (15) vacuum chamber.

each neutron bunch is about $40 \mathrm{~cm}$ while the TPC is $96 \mathrm{~cm}$ long. This setup enables us to define a fiducial time, during which each neutron bunch is completely inside the sensitive region of the TPC. As a result, $4 \pi$ solid angle acceptance is achieved for both the beta-decays and ${ }^{3} \mathrm{He}(n, p){ }^{3} \mathrm{H}$ reactions.

The neutron lifetime can be expressed as the ratio of the numbers for the two kinds of events $\left(S_{\beta}\right.$ and $\left.S_{{ }^{3} \mathrm{He}}\right)$ as

$$
\tau_{n}=\frac{1}{\rho \sigma_{0} v_{0}} \frac{S_{{ }^{3} \mathrm{He}} / \varepsilon^{3} \mathrm{He}}{S_{\beta} / \varepsilon_{\beta}},
$$

where $\varepsilon_{\beta}$ and $\varepsilon^{3} \mathrm{He}$ are the detection efficiencies of the respective processes, $\rho$ is the ${ }^{3} \mathrm{He}$ number density in the TPC, and $\sigma_{0}=(5333 \pm 7)$ barn is the cross section of ${ }^{3} \mathrm{He}(n, p){ }^{3} \mathrm{H}$ determined at the thermal neutron velocity $v_{0}=2200 \mathrm{~m} / \mathrm{s}$ [12]. Note that $\sigma_{0} v_{0}$ has replaced the product $\sigma v$, using the assumption that $\sigma$ is inversely proportional to the neutron velocity $v$.

In contrast to the mentioned previous in-flight experiments that relied on detection of decay protons $[9,10]$, our experiment detects the decay electrons. In addition, our detector measures the neutron beam intensity as well as the neutron beta-decay events simultaneously and in the same place. Therefore, the systematic uncertainties related to the detector fiducial volume can be suppressed.

An experiment based on this measurement principle, originally proposed by R. Kossakowski et al., was conducted at Institut Laue-Langevin [13]. The result, $\tau_{n}=$ $878 \pm 27$ (stat.) \pm 14 (sys.) s, was statistically limited due to the low flux of the beam available at that time.

\section{Setup of our experiment at J-PARC}

\subsection{Beamline}

The neutron lifetime experiment is conducted at the BL05 Neutron Optics and fundamental Physics (NOP) beamline, which is one of the 21 neutron beamlines of the Materials and Life Science Experimental Facility (MLF) at J-PARC. We use the polarized beam branch at BL05, whose the polarization was measured to be $94 \sim 97 \%$ [14]. The expected neutron particle flux is $(4.0 \pm 0.3) \times 10^{7}$ neutrons $/\left(\mathrm{cm}^{2} \cdot \mathrm{s}\right)$ at the designed MLF operation power of $1 \mathrm{MW}$ [15]. The neutron beam can be switched on and off by a shutter made of a ${ }^{6} \mathrm{LiF}$ plate.
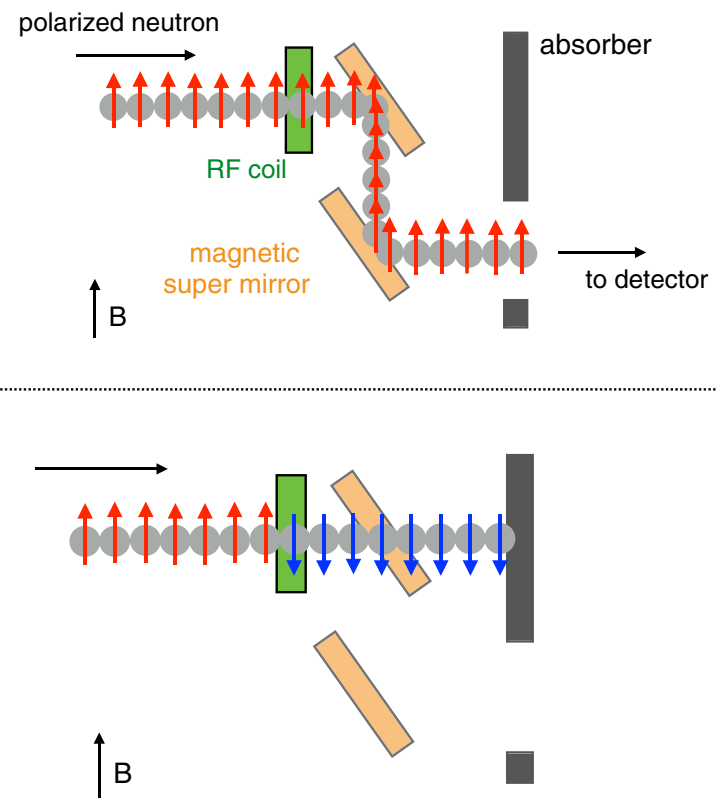

Figure 2. Principle of the SFC operation. The neutron beam passes through the SFC when no current is applied at the RF coil (Top), and is absorbed when RF current is applied (Bottom).

\subsection{Spin flip chopper}

The SFC forms neutron bunches from a polarized neutron beam, employing the basic principle described in Fig. 2. It is composed of radio-frequency (RF) coils and magnetic super mirrors, surrounded by a lead shield. A magnetic field of $1 \mathrm{mT}$ is applied in the vertical direction uniformly throughout the SFC to maintain the neutron polarization. Application of RF current rotates by $180^{\circ}$ the polarization of the neutrons passing through the device. The magnetic super mirror selectively reflects only non-flipped neutrons that are subsequently guided further downstream. By switching the RF current on and off, neutron bunches with arbitrary lengths can be formed within a single pulse from the spallation source.

\subsection{Time projection chamber}

In this experiment, a TPC with a two-dimensional Multi Wire Proportional Chamber (MWPC), which was specially developed in our group [11], was used to detect both 


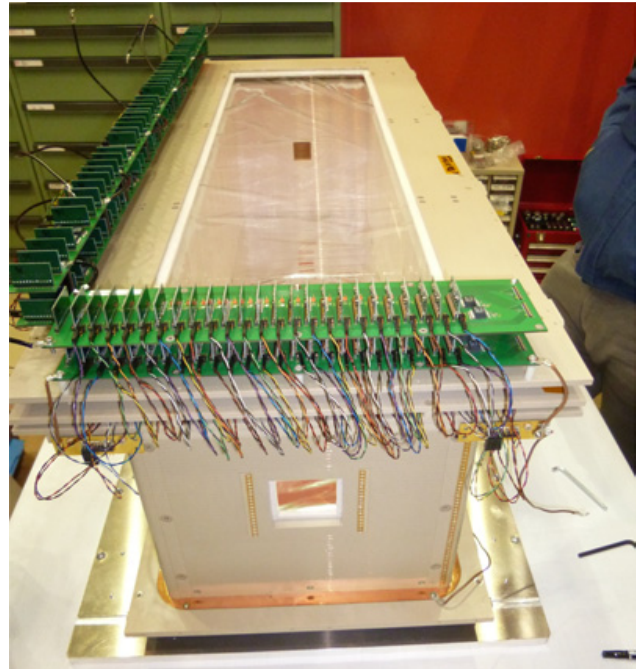

Figure 3. Picture of the TPC [16].

Table 1. Basic parameters of the TPC [11].

\begin{tabular}{|l|l|}
\hline size & $300 \mathrm{~mm} \times 300 \mathrm{~mm} \times 960 \mathrm{~mm}$ \\
anode wire $(24 \mathrm{ch})$ & $\phi 20 \mu \mathrm{m}, 1720 \mathrm{~V}$ \\
field wire $(24 \mathrm{ch})$ & $\phi 50 \mu \mathrm{m}, 0 \mathrm{~V}$ \\
cathode wire $(40 \mathrm{ch} \times 2)$ & $\phi 50 \mu \mathrm{m}, 0 \mathrm{~V}$ \\
electric field & $300 \mathrm{~V} / \mathrm{cm}$ (vertical direction) \\
drift velocity & $1.0 \mathrm{~cm} / \mu \mathrm{s}$ \\
multiplication factor & $5 \times 10^{4}$ \\
basic gas composition & ${ }^{4} \mathrm{He}(85 \mathrm{kPa}), \mathrm{CO}_{2}(15 \mathrm{kPa}),{ }^{3} \mathrm{He}$ \\
& $(100 \mathrm{mPa})$ \\
\hline
\end{tabular}

neutron beta-decay and ${ }^{3} \mathrm{He}(n, p){ }^{3} \mathrm{H}$. A picture of the TPC is shown in Fig. 3. Its size is $30 \mathrm{~cm} \times 30 \mathrm{~cm} \times 96 \mathrm{~cm}$, and it is filled with $\mathrm{He}$ and $\mathrm{CO}_{2}$ as operation gas. The TPC is made of Polyether ether ketone (PEEK) in order to suppress the background from radioactive isotopes. The inner walls are covered by ${ }^{6} \mathrm{LiF}$ plates, which capture neutrons with little prompt $\gamma$-ray emission. A lead shield reduces ambient $\gamma$-ray background. The whole setup, except at the bottom, is surrounded by fourteen plastic scintillators which serve for identification of cosmic ray events. The detailed specification of the TPC is listed in Table 1.

Ionization electrons from a charged track drift upwards by a uniform electric field of $300 \mathrm{~V} / \mathrm{cm}$, causing an avalanche multiplication in the MWPC region located at the top of the TPC. The MWPC is composed of three wire layers. In the middle layer, 24 anode wires of $20 \mu \mathrm{m}$ diameter and 24 field wires of $50 \mu \mathrm{m}$ diameter are aligned alternately with an interval of $6 \mathrm{~mm}$. In the above and below layers, 162 cathode wires of $50 \mu \mathrm{m}$ diameter are aligned at $6 \mathrm{~mm}$ interval perpendicular to the anode and field wires, and every four cathode wires in the same layer are electrically connected and read out as a single channel.

\subsection{Data acquisition system}

A schematic diagram of the data acquisition system is shown in Fig. 4. The signals of the TPC and the cosmic ray veto counters were recorded by the Front-end INstrumentation Entity for Sub-detector Specific Electronics (FINESSE) modules in the COmmon Pipelined

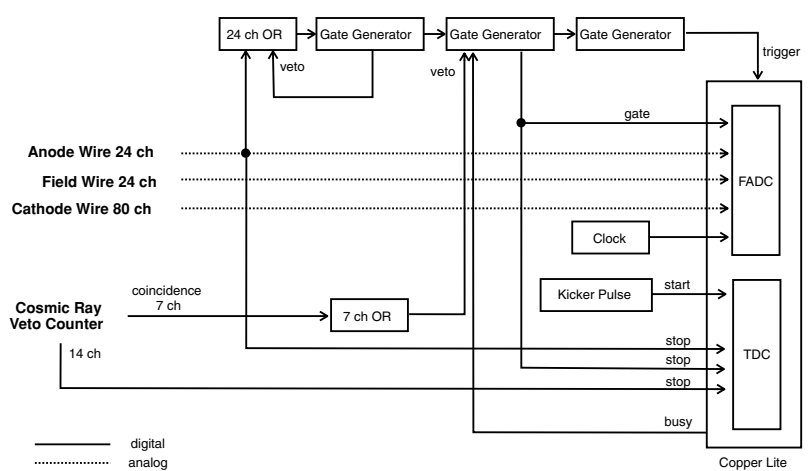

Figure 4. Schematic diagram of the DAQ circuit [16]. The ADC and the TDC operating in the COPPER system [17] are used for taking the data. Wire waveforms of the TPC are recorded by the flash-ADC, whereas timing of waveforms, kicker pulse, and cosmic ray veto counters are recorded by the TDC.

Table 2. Physics data set.

\begin{tabular}{|c|cc|c|cc|}
\hline Period & Start & End & $\begin{array}{c}\text { Operation } \\
\text { power }\end{array}$ & $\begin{array}{c}\text { Beam } \\
\text { data }\end{array}$ & $\begin{array}{c}\text { Neutrons } \\
\text { entering TPC }\end{array}$ \\
\hline 2014A & $2014 / 5 / 27$ & $2014 / 6 / 3$ & $300 \mathrm{~kW}$ & 34.2 hours & $2.3 \times 10^{10}$ \\
$2015 \mathrm{~A}$ & $2015 / 4 / 26$ & $2015 / 5 / 4$ & $500 \mathrm{~kW}$ & 15.8 hours & $2.0 \times 10^{10}$ \\
$2016 \mathrm{~A}$ & $2016 / 4 / 16$ & $2016 / 6 / 14$ & $200 \mathrm{~kW}$ & 224 hours & $1.2 \times 10^{11}$ \\
\hline Combined & & & & 274 hours & $1.6 \times 10^{11}$ \\
\hline
\end{tabular}

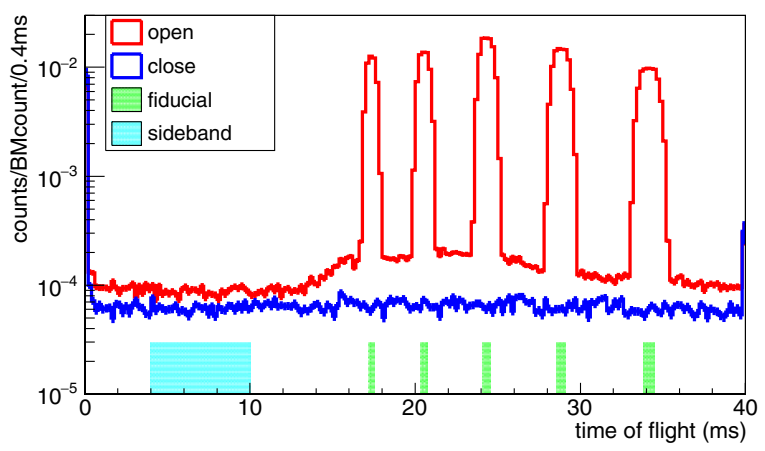

Figure 5. ${ }^{3} \mathrm{He}(n, p){ }^{3} \mathrm{H}$ reaction events in the TPC in correlation to the neutron TOF. The red and blue histograms represent the shutter open and close data, respectively. The vertical axis is normalized by the counts of the neutron beam flux monitor. Each of the five green bands represents the fiducial time, in which a neutron bunch is completely inside the TPC sensitive region. Their durations are $0.33 \mathrm{~ms}, 0.39 \mathrm{~ms}, 0.46 \mathrm{~ms}, 0.54 \mathrm{~ms}$, and $0.64 \mathrm{~ms}$, in ascending order of TOF. The light blue band represents a sideband region (in the TOF range $4 \sim 10 \mathrm{~ms}$ ), during which no neutron bunch is inside the TPC. The time $t=0$ defines the moment at which the proton beam hits the spallation target in the MLF. The spallation process causes a large background, which can be seen as the peaks at $t=0 \mathrm{~ms}$ and $t=40 \mathrm{~ms}$.

Platform for Electronics Readout (COPPER) system [17]. On 5 COPPER boards, 16 FINESSE ADC modules and 2 FINESSE TDC modules were implemented. Waveforms of 24 anode wires, 24 field wires, and $40 \times 2$ cathode wires were digitized by the ADC modules. The timing information of TPC signals, veto counters, and a kicker pulse, which represents the time of producing the neutron beam, was digitized by the TDC modules. All digitized data was sent to a computer through ethernet cables. The 

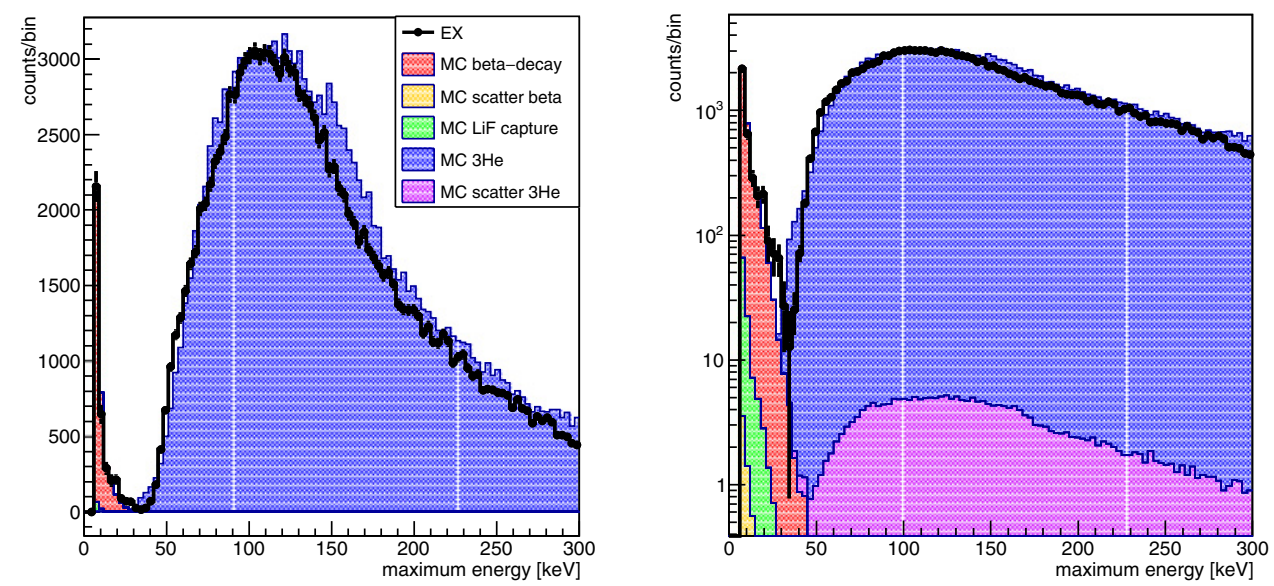

Figure 6. Monte-Carlo simulated distributions of energy deposits sensed by the field wires for the different processes in a linear scale (Left) and a logarithmic scale (Right), together with the experimental data shown as black dots. One can see a clear separation of the beta-decay events from the ${ }^{3} \mathrm{He}(n, p)^{3} \mathrm{H}$ reaction events. The threshold is set at $25 \mathrm{keV}$. The "scatter beta" denotes neutron beta-decays after scattering by the TPC gas, and the "scatter $3 \mathrm{He}$ " denotes captures of scattered neutrons by ${ }^{3} \mathrm{He}$. The events denoted by "LiF capture" are due to ionization by $\gamma$-rays emitted after neutron capture in the LiF plates.
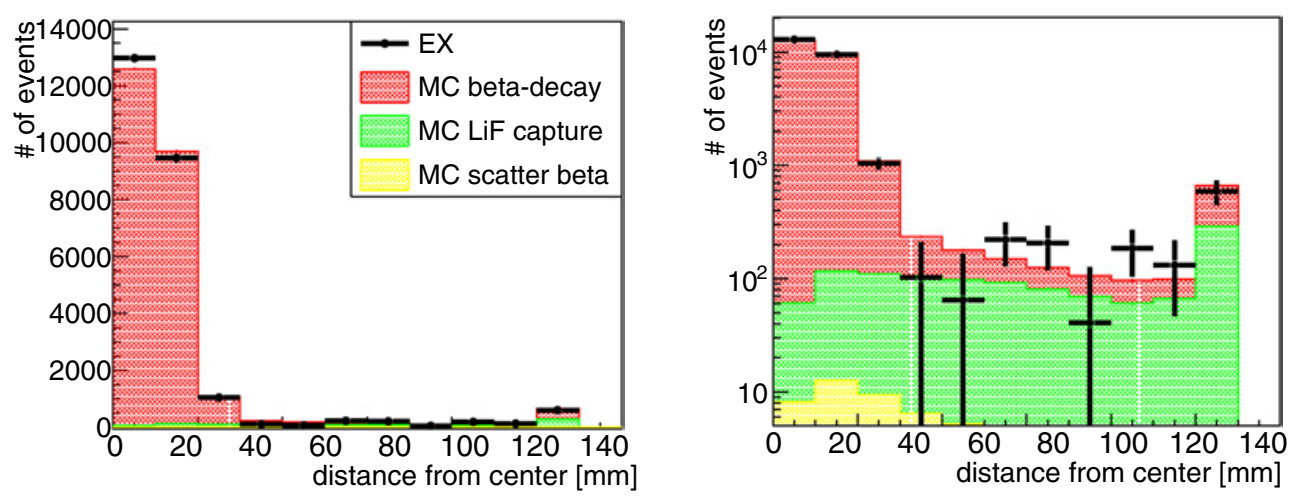

Figure 7. Distribution of the "distance from center" parameter in a linear scale (Left) and a logarithmic scale (Right). The event types shown are the same as in Fig. 6.

trigger signal for taking the data was sent to the five COPPER boards when any of the 24 anode wires in the TPC exceeded a threshold voltage of $20 \mathrm{mV}$, corresponding to $200 \mathrm{eV}$ energy deposit in the TPC. During the data transfer time, which was typically $100 \mu$ s per trigger signal, the COPPER modules gave a busy signal and any additional trigger signal was rejected. The trigger signal was also vetoed for $70 \mu \mathrm{s}$ when any set of 7 plastic scintillators gave signals in coincidence with the TPC signal, in order to avoid triggering to cosmic ray events.

\section{Analysis}

\subsection{Dataset}

Table 2 shows the data set of the neutron lifetime measurement acquired between 2014 and 2016. During a total of 11 days, $1.6 \times 10^{11}$ neutrons entered the TPC. The data was taken for six different values of partial pressure of the ${ }^{3} \mathrm{He}$ gas in the range of $50 \mathrm{mPa}$ to $200 \mathrm{mPa}$.

\subsection{Background subtraction using time of flight}

Figure 5 shows a distribution of ${ }^{3} \mathrm{He}(n, p)^{3} \mathrm{H}$ reaction events correlated with the neutron time of flight (TOF), for the data with open and closed shutter, respectively. Five peaks formed by the SFC within each pulse from the spallation source can be clearly seen. The constant background, such as cosmic rays and environmental radiation, can be estimated using the data in the sideband region.

\subsection{Energy separation}

A typical beta-decay event deposits only a few $\mathrm{keV} / \mathrm{cm}$ in the TPC, whereas the neutron capture reaction deposits a few hundred keV. Accordingly, these two types of events can be well separated using the maximum energy deposited per wire among all field wires. Note that this parameter is more sensitive for the separation than the total energy deposited in all the 24 field wires. Figure 6 shows distributions of this parameter, in which a threshold energy is set at $25 \mathrm{keV}$.

\subsection{Scattering event analysis}

Neutrons are scattered by gas molecules in the TPC with a probability of about $1 \%$. Most of them are captured by ${ }^{6} \mathrm{LiF}$ plates covering the TPC wall. Prompt $\gamma$-rays from the plates cause Compton scatterings in the TPC, which become background for beta-decay. A useful characteristics for distinction is that the ionization tracks from background events occur uniformly in the TPC, whereas those from the beta-decays come from locations 
Table 3. Result of a single neutron lifetime run and list of parameters entering Eq. (1) for analysis (very preliminary). Experimental data were taken in 2016. The last column describes the respective contribution of each quantity to the uncertainty on $\tau_{n}$.

\begin{tabular}{|c|c|c|c|}
\hline Quantity & Value & Units & Uncertainty on $\tau_{n}$ \\
\hline$S_{3_{\mathrm{He}}}$ & $358101 \pm 635$ (stat.) ${ }_{-210}^{+408}$ (sys.) & events & 0.18 (stat. $)_{-0.06}^{+0.11}$ (sys.) \\
\hline$S_{\beta}$ & $14432 \pm 373$ (stat.) ${ }_{-181}^{+85}$ (sys.) & events & 2.59 (stat. $)_{-1.26}^{+0.59}$ (sys.) \\
\hline$\epsilon_{3 \mathrm{He}}$ & $99.99_{-0.00}^{+0.01}$ (sys.) & $\%$ & ${ }_{-0.00}^{+0.01}$ (sys.) \\
\hline$\epsilon_{\beta}$ & $93.92_{-0.81}^{+0.61}$ (sys.) & $\%$ & ${ }_{-0.86}^{+0.65}$ (sys.) \\
\hline$\rho$ & $2287_{-10}^{+10}$ (sys.) & $10^{16}$ atoms $/ \mathrm{m}^{3}$ & ${ }_{-0.42}^{+0.42}$ (sys.) \\
\hline$\sigma_{0}$ & $5333^{2} 7$ (sys.) & $10^{28} \mathrm{~m}^{2}$ & 0.13 (sys.) \\
\hline$v_{0}$ & 2200 & $\mathrm{~m} / \mathrm{s}$ & exact \\
\hline \hline$\tau_{n}$ & $868.8 \pm 22.5$ (stat.) ${ }_{-9.9}^{+12.9}$ (sys.) & $\mathrm{s}$ & 2.59 (stat.) $)_{-1.14}^{+1.49}$ (sys.) \\
\hline
\end{tabular}

Table 4. Neutron lifetime results from all the data taken between 2014 and 2016 (very preliminary).

\begin{tabular}{c|c|c} 
ID & date & $\tau_{n}[\mathrm{~s}]$ \\
\hline 1 & $2014 / 5$ & $943.5 \pm 25.3_{-11.1}^{+20.6}$ \\
2 & $2015 / 4$ & $904.4 \pm 19.6_{-7.6}^{+13.2}$ \\
3 & $2016 / 4$ & $899.5 \pm 45.5_{-11.9}^{+11.7}$ \\
4 & $2016 / 4$ & $887.7 \pm 22.2_{-10.4}^{+15.5}$ \\
5 & $2016 / 5$ & $879.2 \pm 23.5_{-11.7}^{+11.7}$ \\
6 & $2016 / 6$ & $868.6 \pm 22.5_{-9.9}^{+12.9}$ \\
\hline \multicolumn{2}{c}{ Combined } & $895.7 \pm 9.8$ (stat.) \\
\hline
\end{tabular}

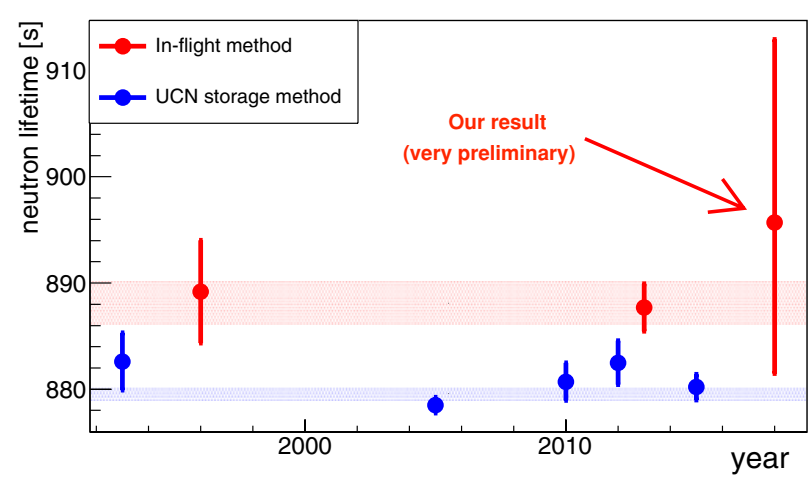

Figure 8. Our result of the neutron lifetime along with previous measurements [3]. The average of the two most recent results using the in-beam method (red) is $(888.0 \pm 2.0)$ s $[9,10]$, while that using UCN storage (blue) is $(879.6 \pm 0.6) \mathrm{s}$ [4-8]. The discrepancy of the two averages is $8.4 \mathrm{~s}(4.0 \sigma)$.

intersected by the neutron beam. A parameter called "distance from center", which represents the distance between the track origin and the beam axis, is used for the separation. Figure 7 shows the distribution, and the cut threshold was set at 4 wires (corresponding to $4.8 \mathrm{~cm}$ from the beam center position). The background contamination in the signal region $(\sim 5 \%)$ was evaluated by a Monte Carlo simulation. The uncertainty of the contamination is not yet completely evaluated, so that the present result is still preliminary.

\subsection{Systematic uncertainties}

In this section, some of the dominant systematic uncertainties are described.
- Pileup events:

During the data taking time of $100 \mu$ s per trigger signal, there is a possibility of recording multiple events, which needs to be corrected for. The expected amount of these pileup events is calculated from the time window width and the event rate of the respective signal events. The uncertainty is evaluated to be $+0.4 /-1.2 \%$ for the number of the neutron betadecay events, and currently dominates the total systematic uncertainty.

- ${ }^{3} \mathrm{He}$ contamination in ${ }^{4} \mathrm{He}$ gas:

The ${ }^{4} \mathrm{He}$ component of the operation gas of the TPC contains a small amount of ${ }^{3} \mathrm{He}(\sim 0.1 \mathrm{ppm})$. It contributes about $10 \%$ to the total ${ }^{3} \mathrm{He}$ pressure in the TPC and therefore needs to be measured precisely, for which we used a mass spectrometer [18]. The stability of this spectrometer led to a systematic uncertainty of $0.3 \%$ on the total ${ }^{3} \mathrm{He}$ number density in the TPC.

- Signal separation:

As said in Sect. 4.3, the beta-decay events and the ${ }^{3} \mathrm{He}(n, p){ }^{3} \mathrm{H}$ reaction events are separated based on the energy deposit in the TPC. The separation efficiency is limited by the energy calibration accuracy of the TPC $(5 \sim 9 \%)$, causing a systematic effect of $+0.5 /$ $-0.7 \%$.

\section{Result}

The neutron lifetime is evaluated independently for six runs performed at different partial pressure of ${ }^{3} \mathrm{He}$. Table 3 lists the values of all entries to Eq. (1) for one of these runs performed in 2016. The results of all six runs performed between 2014 and 2016 are listed in Table 4. Note that these values and uncertainties are very preliminary. They are consistent with each other. The combined value is $896 \pm 10$ (stat.) ${ }_{-10}^{+14}$ (sys.) s and is shown along with previous results of other groups in Fig. 8.

\section{Summary and discussion}

A new neutron lifetime measurement is currently conducted at J-PARC. A spin flip chopper is used to form neutron bunches from the polarized neutron beam to increase the signal-to-noise ratio. A TPC is used as a 


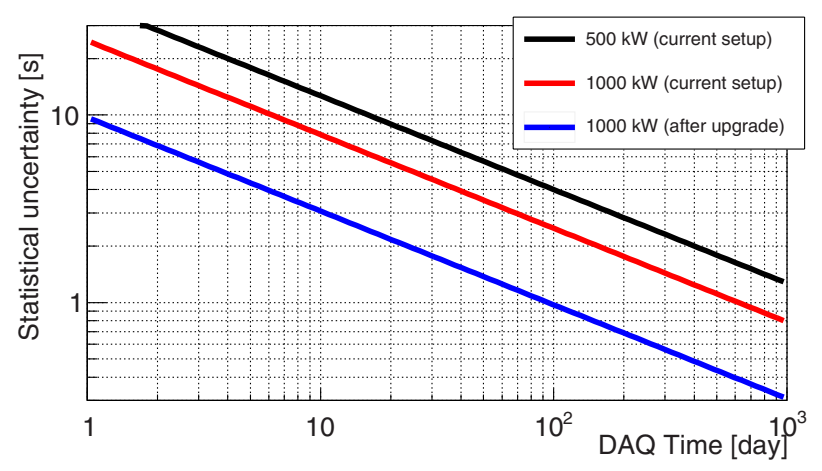

Figure 9. Expected statistical uncertainties as a function of the total data acquisition time. After the SFC upgrade, the neutron beam intensity is estimated to increase by 5.3 times.

beta-decay detector, which also counts the ${ }^{3} \mathrm{He}(n, p){ }^{3} \mathrm{H}$ reaction events simultaneously for the flux evaluation. Our first preliminary result, using the data between 2014 and 2016 , is $896 \pm 10$ (stat. $)_{-10}^{+14}$ (sys.) s. The precision is still ten times worse than our precision goal of $1 \mathrm{~s}$, and several upgrades are planned to improve both the statistical and systematic uncertainties.

The currently employed SFC limits the beam size in the TPC to $2.5 \mathrm{~cm} \times 2 \mathrm{~cm}$. An upgraded device will increase the beam size to $10 \mathrm{~cm} \times 3 \mathrm{~cm}$, corresponding to a 5.3 times larger neutron beam intensity. As shown in Fig. 9, a 100-days measurement will then yield $1 \mathrm{~s}$ statistical uncertainty. Since the background originating from neutron scattering causes one of the biggest uncertainties, we also plan to reduce the operation gas pressure of the TPC. Complying with this, a new type of preamplifier with low power consumption is currently being developed and tested in our group.

\section{References}

[1] J. Chadwick, Proc. R. Soc. London A: Math. Phys. Eng. Sci. 136, 692 (1932)

[2] M. Pospelov, J. Pradler, Ann. Rev. Nucl. Part. Sci. 60, 539 (2010)

[3] M. Tanabashi et al., (Particle Data Group) Phys. Rev. D 98, 030001 (2018)

[4] W. Mampe, L.N. Bondarenko, V.I. Morozov, Yu. N. Panin, A. I. Fomin, JETP Lett. 57, 82 (1993)

[5] A. Serebrov et al., Phys. Lett. B 605, 72 (2005)

[6] A. Pichlmaier, V. Varlamov, K. Schreckenbach, P. Geltenbort, Phys. Lett. B 693, 221 (2010)

[7] A. Steyerl, J.M. Pendlebury, C. Kaufman, S.S. Malik, A.M. Desai, Phys. Rev. C 85, 065503 (2012)

[8] S. Arzumanov et al., Phys. Lett. B 745, 79 (2015)

[9] J. Byrne, P.G. Dawber, Europhys. Lett. 33, 187 (1996)

[10] A.T. Yue et al., Phys. Rev. Lett. 111, 222501 (2013)

[11] Y. Arimoto et al., Nucl. Instrum. Methods Phys. Res. Sect. A 799, 187 (2015)

[12] S.F. Mughabghab, Atlas of Neutron Resonances: Resonance Parameters and Thermal Cross Sections, $\mathrm{Z}=1-100$ (5th edition), (2006)

[13] R. Kossakowski, P. Grivot, P. Liaud, K. Schreckenbach, G. Azuelos, Nucl. Phys. A 503, 473 (1989)

[14] T. Ino et al., Physica B: Condensed Matter 406, 2424 (2011)

[15] K. Mishima, Neutron network news (Hamon) 25, 156 (2015)

[16] N. Nagakura, Master's thesis, The University of Tokyo (2016)

[17] Y. Igarashi et al., Nucl. Sci. IEEE Trans. 52, 2866 (2005)

[18] H. Sumino, K. Nagao, K. Notsuji, J. Mass Spectrom. Soc. Japan 49, 61 (2001) 